\title{
Physical examination, special tests and investigations for the diagnosis of WRMSD
}

\author{
Deepak Sharan ${ }^{1}$. \\ ${ }^{1}$ RECOUP Neuromusculoskeletal Rehabilitation Centre, Bangalore, INDIA.
}

\begin{abstract}
Physical examination will enable the clinician to link the information obtained from history and physical findings to make the precise diagnosis, patient education, to design an effective treatment plan and optimum rehabilitation prescription.
\end{abstract}

Keywords: Diagnosis, Orthopaedic Special Tests, Neural Tension Testing

\section{Classification of WRMSD's}

The WRMSD's are classified in to two broad categories; 1) Specific and 2) Non-Specific (Patient presents with pain, discomfort or functional impairment but no specific diagnosis or pathology can be ascertained).

\subsection{Specific WRMSD's}

1.1.1 Tendon-related conditions

1.1.2 Nerve-related conditions

1.1.3 Circulatory/vascular conditions

1.1.4 Joint-related conditions

1.2 Non-Specific WRMSD's

1.2.1 Myofascial pain syndrome (MPS)

1.2.2 Fibromyalgia syndrome (FMS)

1.2.3 Complex regional pain syndrome (CRPS)

\section{Diagnostic criteria}

The criteria used for the diagnosis of individual patients in the office setting include both symptoms (i.e., the nature of a patient's present complaints) and clinical signs (i.e., positive findings, according to some definition, in a physical examination). Accurate diagnosis helps to determine the most appropriate treatment and protects the patient from treatments that are ineffective, unwarranted, or unnecessarily invasive.

In the RECOUP's on-site clinics the employees are diagnosed based on the musculoskeletal complaints from the employee history (the pain history, the occupational, personal, family and medical history), medical examination including Observation (posture analysis, swelling, skin colour, Active Range of Motion), Palpation (muscular strength examination, texture examination, passive range of motion, trigger point and tender point examination). Sometimes Orthopaedic Special Test and Neural Selective Tension Test are utilised to confirm the conditions. These tests help to differentiate the other conditions from the exact diagnosis.

\subsection{Observation}

The observation includes different working posture (with or without equipment) as faulty postures are one of the major causes of overuse injuries. Clinician should also look out for any evidence of deformity, asymmetry, bruising, swelling, skin colour and temperature changes and muscle wasting. Active range of motion (ROM) assessment helps clinician to look for any restriction of ROM, the onset of pain at particular range and the presence of abnormal movement patterns.

\subsection{Palpation}

It is the critical part of the examination and thorough knowledge of surface anatomy optimises its value in making tissue specific diagnosis. Resistance, muscle spasm, trigger points, palpable muscle band, tender points are the properties of the soft tissue which needs to be palpated. Muscle palpation includes muscle tone assessment, muscle length and imbalance. While assessing muscle strength and 
passive ROM, compare it with the unaffected side. As these both may be present due to injury or may act as a predisposing factor towards injury.

\subsection{Orthopaedic Special tests}

It is helpful to reproduce the patient's symptoms and orthopaedic special test are helpful in order to reproduce the patient's symptom/s and to determine the structures are injures or at fault. The special tests for both specific and non-specific WRMSD's are mentioned later in this paper.

\subsection{Neural Tension Testing}

Advances in the understanding of neural tension have led to understanding of why restrictions of the normal mechanics of joints and muscles can contribute to restriction of normal mechanics of nervous system and pain occurrence in chronic overuse injuries and pain syndromes. It contains few successive movements which makes variations in nerve length in order to produce systematic increase in neural tension which ultimately reproduces the patient's symptoms like pain, pins and needles or numbness. Other investigation techniques like x-rays, blood test (uric acid level, RA factors, thyroid etc.), CT scan, MRI, Bone densitometry etc are relevant for the diagnosis of WRMSD's.

\section{Diagnostic Criteria of Specific WRMSD's}

Specific WRMSD's are diagnosis based on three factors 1) Time rule: Symptoms present now or at least 4 days during the last 1 week, 2) Symptoms: Intermittent pain/ache in that particular region and 3) Signs: Provocation of symptoms (physical examination and special test). Special tests for specific WRMSD's are mentioned below.

\subsection{Tendon-related conditions}

3.1.1 Finkelstein's Test

3.1.2 Cozen's Test

3.1.3 Golfer's Elbow Test

\subsection{Nerve-related conditions}

\subsubsection{Tinel's test [1]}

Examiner percusses over palm from proximal palmar crease to distal wrist crease. Positive if symptoms are elicited in distribution of median nerve.

3.2.2 Phalen's test [1]

Patient places dorsal aspects of hands together, maintaining maximal wrist flexion for 60 seconds.
Test is positive if symptoms are elicited in distribution of median nerve.

\subsection{Circulatory/vascular conditions}

\subsubsection{Adson's test [3]}

The patient begins the test with the head laterally rotated to the side of the symptoms and extended. The patient then abducts the involved arm and breaths in deeply. A positive obliterates radial pulse and reproduces symptoms.

3.3.2 Allen's test

3.3.3 Roos' test [3]

Patient opens and closes his or her hands for 1-3 minutes with elbows bent and arms abducted to $90^{\circ}$ and externally rotated in an attempt to reproduce the symptoms.

\section{Diagnostic criteria of non-specific WRMSD's}

Non-Specific WRMSD's are diagnosed based on typical individual factors. That is Myofascial Pain Syndrome by Simon's major and minor criteria (P S Ajeesh, et. al, 2010 and Simons, D.G., 1999), Fibromyalgia by American college of Rheumatology (ACR) criteria (Wolfe .F et al., 2010) as mentioned below and CRPS based on history, signs, symptoms and associated problems.

\subsection{Simon's Major Criteria}

4.1.1 Localised spontaneous pain.

4.1.2 Spontaneous pain or altered sensations in expected referred pain area for given multiple trigger points (MTrP)

4.1.3 Taut, palpable band in accessible muscle.

4.1.4 Exquisite, localised tenderness in precise point along taut band

4.1.5 Some measurable reduced movement range

\subsection{Simon's Minor Criteria}

4.2.1 Reproduction of spontaneously perceived pain and altered sensations by pressure on MTrP

4.2.2 Elicitation of local twitch response of muscular fibers by transverse "snapping" palpation or by needle insertion into MTrP

4.2.3 Pain relief obtained by muscle stretching or injection of MTrP 


\subsection{The 1990 ACR criteria for the diagnosis of fibromyalgia}

4.3.1 The first is the presence of pain in all 4 quadrants of the body, as well as in the axial skeleton on a more or less continuous basis for at least 3 months

4.3.2 The second criterion is the presence of at least 11 of 18 anatomically specific tender points

\section{References}

[1] J. Cleland, Wrist and Hand, in: Orthopaedic Clinical Examination: An evidence-Based Approach for Physical Therapists, Saunders Elsevier, Philadelphia, 2005, pp. 445-500.
[2] P. Brukner and K. Khan, Principles of Diagnosis: Clinical Assessment, in: Clinical Sports Medicine, $3^{\text {rd }}$ ed., McGraw-Hill, Sydney, London, 2006, pp. 108119.

[3] P. Brukner and K. Khan, Shoulder Pain, in: Clinical Sports Medicine, W. Ben Kibler and G.A.C. Murrell, $3^{\text {rd }}$ ed., McGraw-Hill, Sydney, London, 2006, pp. 243288 .

[4] J. Cleland, Wrist and Hand, in: Orthopaedic Clinical Examination: An evidence-Based Approach for Physical Therapists, Saunders Elsevier, Philadelphia, 2005, pp. 445-500.

[5] P. Brukner and K. Khan, Principles of Diagnosis: Clinical Assessment, in: Clinical Sports Medicine, $3^{\text {rd }}$ ed., McGraw-Hill, Sydney, London, 2006, pp. 108119 .

[6] P. Brukner and K. Khan, Shoulder Pain, in: Clinical Sports Medicine, W. Ben Kibler and G.A.C. Murrell, $3^{\text {rd }}$ ed., McGraw-Hill, Sydney, London, 2006, pp. 243288. 